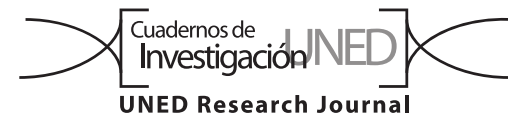

\title{
Selección de vacas Jersey y Holstein durante la lactancia según características fenotípicas: producción y reproducción
}

\author{
Juan MI. Villalobos Alvarez'1 \& Rodolfo WingChing-Jones² \\ 1. Impulso Pecuario, Alajuela, Costa Rica; impesajm@yahoo.com \\ (D) https://orcid.org/0000-0002-2415-6515 \\ 2. Universidad de Costa Rica, Escuela de Zootecnia, Centro de Investigación en Nutrición Animal, Costa Rica; rodolfo.wingching@ucr.ac.cr \\ (D) https://orcid.org/0000-0002-8009-2210
}

Recibido 06-XI-2018 • Corregido 04-III-2019 • Aceptado 01-IV-2019

DOI: https://doi.org/10.22458/urj.v11i3.2579

\begin{abstract}
Jersey and Holstein cow selection during lactation according to phenotypic characteristics: production and reproduction". Introduction: associating the physical characteristics of the animals within a production system is a tool to group animals that can adapt to climate, topography, and handling conditions, without an impact on their productivity. Objective: our objective was to evaluate the relationship between 17 physical variables, from 1260 animals of the Holstein and Jersey breeds, with their productivity in milk production systems located on the foothills of the Poás Volcano in Alajuela, Costa Rica, during the years 2013 and 2014. Methods: the nutritional and sanitary conditions of the studied farms were very similar. To this end we reconciled information of the daily production per cow, the lactation production adjusted to 305 days, the interval between calves, open days, postpartum days to the first service, and services for conception. Results: the height, physical capability, characteristics of the milk, strength, and rump angle are the parts of the cow that have the most influence on their reproductive performance. The conformation of the udder has no influence on the reproduction parameters, there are no statistical differences between the different scores for the characteristics of the udder and the reproductive parameters, but some trends of both breeds can be mentioned. The udder is more related to milk production than the legs and the hooves. Conclusions: the results of this research are exploratory, but they highlight variables that help define a voluntary selection program of Holstein or Jersey animals during the lactation period, which will help obtain animals that can adapt to the handling, environmental, nutritional, and sanitary characteristics of each system.
\end{abstract}

Key words: milk production, lineal measuring, calving interval, dairy character, udder, hooves.
RESUMEN: Introducción: asociar las características físicas de los animales en un sistema de producción es una herramienta que permite agrupar los animales que se adaptan a las condiciones de clima, topografía y manejo, sin afectar su productividad. Objetivo: nuestro objetivo fue evaluar la relación de 17 variables físicas de 1260 animales de las razas Holstein y Jersey; con su productividad en sistemas de producción de leche ubicados en las faldas del Volcán Poás, Alajuela Costa Rica durante los años 2013 y 2014. Metodología: las fincas evaluadas presentaron similitud en el manejo de los animales, en la parte nutricional y en el aspecto sanitario. Para tal fin, recopilamos información de la producción diaria por vaca, la producción por lactancia ajustada a 305 días, el intervalo entre partos, los días abiertos, los días post parto al primer servicio y los servicios por concepción. Resultados: la estatura, la capacidad corporal, el carácter lechero, la fortaleza y el ángulo de anca son las partes de la vaca que más influencia tienen sobre el desempeño reproductivo. La conformación de la ubre no tiene influencia en los parámetros reproductivos, no se obtuvo diferencias estadísticas entre los diferentes puntajes para las características de ubre y los parámetros reproductivos, pero si se puede mencionar tendencias en ambas razas. La ubre está asociada a la producción de leche más que las patas y pezuñas. Conclusión: los resultados de esta investigación son exploratorios, pero permiten visibilizar variables para definir un programa de selección voluntario de animales Holstein o Jersey durante el periodo de lactancia, que permita obtener animales que se adapten según las características de manejo, ambientales, nutricionales y sanitarias de cada sistema.

Palabras clave: producción de leche, evaluación lineal, intervalo entre partos, carácter lechero, ubre, pezuñas. 
La relación de las características físicas de las vacas lecheras con su producción permite escoger un modelo de animal que sea adecuado al sistema, donde se adapte a un medio ambiente específico, produzca la mayor cantidad de leche con la menor cantidad de problemas, por el mayor tiempo posible, para así optimizar la rentabilidad. De tal manera, la descripción física del tipo de animal para la producción de leche permite solventar la especulación de las características físicas que debe tener un semoviente para ser una unidad productora rentable de leche. La conformación deseable de una vaca lechera debe incluir las características físicas asociadas con alta producción de leche por un largo periodo de tiempo, que le permita tener una vida productiva libre de problemas (Stamschror, Seykora, \& Hansen, 2000). En ganado Holstein se especifican 17 variables funcionales agrupadas en características fenotípicas que son indicativos del bienestar de la vaca lechera y que forman parte de los diferentes índices de selección (Holstein Association, 2012). Dentro de ese grupo de índices se encuentran los que miden eficiencia reproductiva, salud, resistencia a enfermedades, ingesta de alimentos y longevidad (Boettcher, 2005). Los programas de selección de los animales están diseñados para aumentar los promedios de producción de leche por vaca, los cuales generan resultados que superan a la población evaluada. De forma involuntaria, las características que favorecen la funcionabilidad de los animales y disminuyen los costos de producción están correlacionadas de forma negativa con la producción de leche, como por ejemplo la fertilidad y la salud (Stevenson, 2012).

Se describe en la literatura, una relación entre estas características de tipo de los animales seleccionados y la productividad de este. Por ejemplo, Fatehi, Stella, Shannon y Boettcher (2003), en sistemas de estabulado libre (free stall), informan que las características de patas y pezuñas se ubicaron en un puntaje superior en promedio al ser comparado con el sistema de estabulado con amarras (tie stall), excepto para patas traseras vistas de atrás. Lo anterior lo relacionaron a la cantidad de horas al día en que los semovientes permanecen estabulados, el tipo de piso y tipo de manejo tanto en situaciones de estabulado, como también en los animales que presentan acceso al pastoreo. En Suecia la selección de animales en busca de una menor incidencia de hemorragia de la suela se basa en una mejor conformación de patas y pezuñas, y además aclaran, que esta no es posible, debido a una baja correlación $(0,08$ a 0,27$)$ entre la conformación de las patas y la incidencia de esta (Uggla, Jakobsen,
Bergsten, Eriksson, \& Strandberg, 2008). En países como Holanda, Inglaterra, España y Francia se incluyó la locomoción como una característica física de selección, la cual se mide en los primeros meses de la primera lactancia, debido a que es un indicador del probable desarrollo de enfermedades de la pezuña en su vida productiva (van der Waaij, Holzhauer, Ellen, Kamphuis, \& Jong, 2005).

En el caso de la evaluación del anca, los cambios en su estructura se relacionan con problema reproductivo que enfrentan las vacas, al sugerir que animales con las puntas del isquión más altas que las del íleon tienen fertilidad deficiente (Caraviello, Weigel, \& Giannola, 2004; Sewalem, Kistemaker, Miglior, \& Van Doomaal, 2004). En contradicción a esta afirmación, en vacas Holstein en el Reino Unido, no se encontró evidencia del impacto de la presencia de puntas de anca altas o bajas, sobre la fertilidad (Wall, White, Coffey, \& Brotherstone, 2005). Pero se obtuvo una tendencia en el intervalo entre partos, donde las ancas de ángulos intermedios tienen menor intervalo entre partos, y las vacas con ancas altas poseen en promedio un mayor intervalo entre partos que los animales de anca nivelada y vacas de anca baja (hasta 4 días). Otra variable que se relaciona con vida productiva del semoviente es el sistema mamario, el cual se correlaciona con intervalos entre partos más largos, ya que es de esperar que una buena ubre representa a un animal saludable, sin embargo, los animales con buenas ubres se correlacionan con mayores producciones de leche en lactancias con 305 días (Wall et al., 2005). Un ejemplo de las características de la ubre que impactan la rentabilidad del sistema son los pezones, ya que durante el ordeño se torna la interface entre la glándula mamaria y la pezonera. En relación con esta afirmación, Weiss, Wenfurtner y Bruckmair (2004) informan que el largo de los pezones no presenta correlación con la capacidad de ordeño (canal de ordeño y tasa de flujo de leche). Además, los animales con un tamaño moderado de pezones (no muy largos, no muy cortos), presentaron la mayor vida productiva, contabilizada en meses productivos (Vacek, Stipkova, Newcova, \& Bouska, 2006). Por último, Vacek et al. (2006) concluyen que semovientes con ligamento anterior levemente mayor al promedio (cinco a siete puntos), con ubres traseras muy altas y con el ligamento central más profundo y fuerte; y ubres con profundidad moderada fueron los animales con mayor vida productiva en el sistema de producción.

Por último, el carácter lechero medido como la angularidad que presenta el animal se asocia con la productividad de los animales, variable que se relaciona de forma 
negativa con la condición corporal. Valores de correlación de -0,72 en los Estados Unidos (Dechow, Roger, Klei, \& Lawlort, 2003) y de -0,61 en Dinamarca (Lassen, Hansen, Sorensen, Aamand, Christensen, \& Madsen, 2003), indican que altos puntajes de carácter lechero (alta angulosidad) se asocian con baja condición corporal, lo que podría influir en la incidencia de enfermedades metabólicas (Dechow, Rogers, Sander-Nielsen, Klei, Lawlor, Clay, Freeman, Abdel-Azim, Kuck, \& Schnell, 2004). Según lo descrito, el objetivo de esta investigación, fue explorar la relación entre 17 variables de tipo de animales Jersey y Holstein bajo un ecosistema similar con los indicadores productivos (Producción de leche por lactancia corregida a 305 días y Producción de leche diaria promedio corregida a 305 días) y reproductivos (Intervalo entre partos, número de servicios e intervalo parto-concepción) para definir las variables que permitan categorizar los animales por su productividad y así establecer un criterio de selección voluntario de animales durante su lactancia en la finca.

\section{MATERIALES Y METODOS}

Este es un trabajo exploratorio realizado con información recolectada entre marzo del 2013 a marzo 2014, para determinar asociaciones entre las diferentes fincas, características físicas, la producción y reproducción de vacas Jersey y Holstein en lactancia en el área del Volcán Poás $\left(10^{\circ} 11^{\prime} 51,42^{\prime \prime} \mathrm{N}-84^{\circ} 13^{\prime} 40,68^{\prime \prime O}\right)$ (Cuadro 1). Esta zona presenta una precipitación promedio de $3441 \mathrm{~mm}$ anuales y $17,2^{\circ} \mathrm{C}$ de temperatura promedio anual, además la altitud va de los $1650 \mathrm{msnm}$ hasta los $2600 \mathrm{msnm}$, humedad promedio del $86 \%$, radiación solar de 16,2MJ, brillo solar de 5,2 horas por día (Mena, 2010). Las seis fincas participantes presentan un sistema de pastoreo rotacional en pasto Kikuyo (Kikuyuocloa clandestinum) asociados con trébol blanco (Trifolium spp) cada 28 días. El uso de un alimento balanceado con $16 \%$ de proteína cruda y 1,9Mcal de energía neta de lactancia, en una relación 2,5 a 1 ( $\mathrm{L}$ de leche por Kg de alimento). Además, presentan similitud en el manejo sanitario (programas de vacunas y desparasitaciones) y reproductivo (palpaciones y protocolos de inseminación) del hato.

Técnica de evaluación lineal de las características de conformación y tipo: para la evaluación lineal se utilizó el programa Select Mating Service (SMS) ${ }^{\mathrm{Tm}}$. En donde, se observó de forma individual cada animal a incluir en la investigación, de dicha observación se obtuvo un valor para cada una de las 17 variables a evaluar, distribuidas en seis características físicas según cuerpo (Cuadro 2), tres en patas y pezuñas (Cuadro 3 ) y ocho en el componente de ubre (Cuadro 4), tales valores se califican en una escala de uno al nueve, sin utilizar decimales. Según el valor asignado a cada variable, esta se interpretaba según la información descrita en los cuadros 2, 3 y 4.

Análisis de la información: se analizaron 1260 registros de animales Holstein (315) y Jersey (945) puros con al menos una lactancia, en el caso de registros de animales cruzados o incompletos, estos no se evaluaron. Las variables cualitativas analizadas fueron la finca y la raza del animal. Mientras que, se consideró como variables cuantitativas al intervalo entre partos (IEP), días desde el parto hasta el primer servicio (P1SR), días abierto o intervalo parto concepción (DA o IPC), producción de leche por lactancia corregida a 305 días (P305), servicios por concepción (SC) y promedio de producción de leche por vaca diario (PVD). La información fue analizada por medio del programa PROC GLM y PROC CORR de SAS (2003). Cuando se determinaron diferencias entre medias, estas

\section{CUADRO}

Información productiva y reproductiva promedio de las fincas utilizadas en esta investigación durante marzo de 2013 a marzo de 2014

\begin{tabular}{|c|c|c|c|c|c|c|}
\hline Finca & Raza & $\begin{array}{l}\text { Número de } \\
\text { vacas en ordeño }\end{array}$ & $\begin{array}{c}\text { Producción láctea } \\
\text { promedio } \\
\text { (kg/animal/día) }\end{array}$ & $\begin{array}{l}\text { Periodo parto } \\
\text { al primer servicio } \\
\text { (días) }\end{array}$ & $\begin{array}{l}\text { Periodo abierto } \\
\text { (días) }\end{array}$ & $\begin{array}{c}\text { Intervalo } \\
\text { entre partos } \\
\text { (días) }\end{array}$ \\
\hline 1 & Holstein/Jersey & 132 & 21,10 & 76 & 97 & 377 \\
\hline 2 & Holstein/Jersey & 149 & 24,89 & 66 & 95 & 375 \\
\hline 3 & Jersey & 275 & 27,10 & 60 & 87 & 367 \\
\hline 4 & Holstein/Jersey & 144 & 25,20 & 72 & 115 & 395 \\
\hline 5 & Holstein/Jersey & 118 & 26,81 & 85 & 107 & 387 \\
\hline 6 & Holstein/Jersey & 131 & 25,02 & 75 & 90 & 370 \\
\hline
\end{tabular}


CUADRO 2

Variables ordinales de cuerpo con su puntaje, grado de agrupamiento y su interpretación

\begin{tabular}{|c|c|c|}
\hline Variable & Puntaje & Interpretación \\
\hline \multirow[t]{3}{*}{ Estatura } & 1 a 3 & alta \\
\hline & 4 a 6 & intermedia \\
\hline & 7 a 9 & baja \\
\hline \multirow[t]{3}{*}{ Carácter lechero } & 1 a 4 & tosco \\
\hline & 5 ó 6 & intermedio \\
\hline & 7 a 9 & alto \\
\hline \multirow[t]{3}{*}{ Fortaleza } & 1 a 4 & débil \\
\hline & 5 ó 6 & intermedio \\
\hline & 7 a 9 & fuerte \\
\hline \multirow[t]{3}{*}{ Profundidad corporal } & 1 a 4 & poco profunda \\
\hline & 5 ó 6 & intermedia \\
\hline & 7 a 9 & profunda \\
\hline \multirow[t]{3}{*}{ Amplitud del anca } & 1 a 4 & estrecha \\
\hline & 5 ó 6 & intermedio \\
\hline & 7 a 9 & amplia \\
\hline \multirow[t]{3}{*}{ Ángulo del anca } & 1 a 4 & alto \\
\hline & 5 & intermedio \\
\hline & 6 a 9 & bajo \\
\hline
\end{tabular}

CUADRO 3

Variables ordinales de patas y pezuñas con su puntaje, grado de agrupamiento y su interpretación

\begin{tabular}{ccl} 
Variable & Puntaje & \multicolumn{1}{c}{ Interpretación } \\
Patas vista trasera & 1 a 4 & muy juntas \\
& 5 & intermedio \\
Patas vista lateral & 6 a 9 & bien separadas \\
& 1 a 3 & muy rectas \\
4 a 6 & curva intermedia \\
& 7 a 9 & muy curvas \\
Ángulo de pezuña & 1 a 5 & $\leq 45$ grados \\
& 6 a 9 & $>45$ grados \\
\hline
\end{tabular}

se separaron por medio de la prueba de Duncan (SAS, 2003). Para el modelo de regresión se analizaron los supuestos de normalidad, homocedasticidad y no multicolinealidad, en los datos recolectados y utilizados en esta investigación. En ambas razas se hizo una selección de variables para obtener el modelo que permite optimizar la producción y reproducción del semoviente, según el procedimiento Stepwise, con el software R. Análisis que permitió, obtener una ecuación de predicción para cada raza con las variables que influyen con la productividad de los animales.
Ética, conflicto de intereses y declaración de financiamiento: los autores declaran haber cumplido con todos los requisitos éticos y legales pertinentes, tanto durante el estudio como en el manuscrito; que no hay conflictos de interés de ningún tipo, y que todas las fuentes financieras se detallan plena y claramente en la sección de agradecimientos. Asimismo, están de acuerdo con la versión editada final del documento. El respectivo documento legal firmado se encuentra en los archivos de la revista.

\section{RESULTADOS}

Correlaciones fenotípicas de las características de tipo con variables productivas y reproductivas: las correlaciones obtenidas en esta investigación se presentan en el Cuadro 5 para las razas evaluadas, todas las correlaciones obtenidas son bajas, cercanas a cero y se dan entre las variables de tipo, reproducción y producción analizadas, lo que indica que hay poca asociación directa entre ellas. Se resalta en el Cuadro 5, que las variables que presentan correlaciones mayores a 0,15 , en el caso del ganado Jersey se concentran en el intervalo entre parto, mientras que en los animales Holstein, estos valores se asocian a aspectos reproductivos como productivos. 
CUADRO 4

Variables ordinales de ubre con su puntaje, grado de agrupamiento y su interpretación

\begin{tabular}{|c|c|c|}
\hline Variable & Puntaje & Interpretación \\
\hline \multirow[t]{3}{*}{ Ubre delantera } & 1 a 4 & débil \\
\hline & 5 & intermedia \\
\hline & 6 a 9 & fuerte \\
\hline \multirow[t]{3}{*}{ Altura de ubre trasera } & 1 a 4 & baja \\
\hline & 5 ó 6 & intermedia \\
\hline & 7 a 9 & alta \\
\hline \multirow[t]{3}{*}{ Ancho de la ubre trasera } & 1 a 4 & angosta \\
\hline & 5 & intermedia \\
\hline & 6 a 9 & ancha \\
\hline \multirow[t]{3}{*}{ Ligamento central } & 1 a 4 & débil \\
\hline & 5 & intermedio \\
\hline & 6 a 9 & fuerte \\
\hline \multirow[t]{3}{*}{ Profundidad de ubre } & $1,2,3$ ó 4 & profunda \\
\hline & 5 & intermedia \\
\hline & $6,7,8$ ó 9 & poco profunda \\
\hline \multirow[t]{3}{*}{ Colocación pezones delanteros } & 1 a 4 & separados \\
\hline & 5 & centralizados \\
\hline & 6 a 9 & muy juntos \\
\hline \multirow[t]{3}{*}{ Colocación pezones traseros } & 1 a 4 & abiertos \\
\hline & 5 & centralizados \\
\hline & 6 a 9 & muy juntos \\
\hline \multirow[t]{3}{*}{ Longitud pezones traseros } & 1 a 4 & $<5 \mathrm{~cm}$ \\
\hline & 5 ó 6 & $5-7 \mathrm{~cm}$ \\
\hline & 7 a 9 & $>7 \mathrm{~cm}$ \\
\hline
\end{tabular}

Relación entre las características de cuerpo sobre variables productivas $\mathbf{y}$ reproductivas en animales Jersey y Holstein: en el Cuadro 6, se muestra que la vaca Jersey con más estatura, más fortaleza, más temperamento lechero, mayor profundidad corporal y de ángulo de anca nivelado, es la que mayor producción de leche presentó. Mientras que, las que presentaron menor fortaleza y profundidad corporal, requieren menos días para presentar un parto. En el caso de los animales Holstein, se cuantificaron las mayores producciones de leche en animales con mayor carácter lechero y profundidad corporal. De igual manera que los animales Jersey, a menor calificación de fortaleza, los animales presentaron un menor intervalo entre parto.

Relación entre características de la ubre sobre variables productivas $y$ reproductivas en animales Jersey y Holstein: en la raza Jersey ubres delanteras intermedias, ubre trasera ancha, ligamento central de fortaleza intermedia y pezones delanteros y traseros ubicados debajo de los cuartos favorecen una mayor producción de leche en una lactancia corregida a 305 días. Las demás características de ubre evaluadas no mostraron diferencia significativa $(p<0,05)$ en sus diferentes niveles con respecto a las variables productivas (Cuadro 7). En el caso del intervalo entre parto, se determina que el ancho de la ubre (angosta o ancha) y el ligamento central (débil o fuerte) presentan efectos en disminuir los días entre partos, en relación con variables intermedias para estas mismas variables. Para la raza Holstein, animales con ubres anchas o angostan permiten registrar una mayor producción de leche a 305 días, de igual manera que animales con ligamentos fuertes o débiles. En el caso de la profundidad de la ubre, animales con ubres más profundas producen entre 645,1 y 1039,5L de leche de más por lactancia que animales con poca profundidad de ubre. 


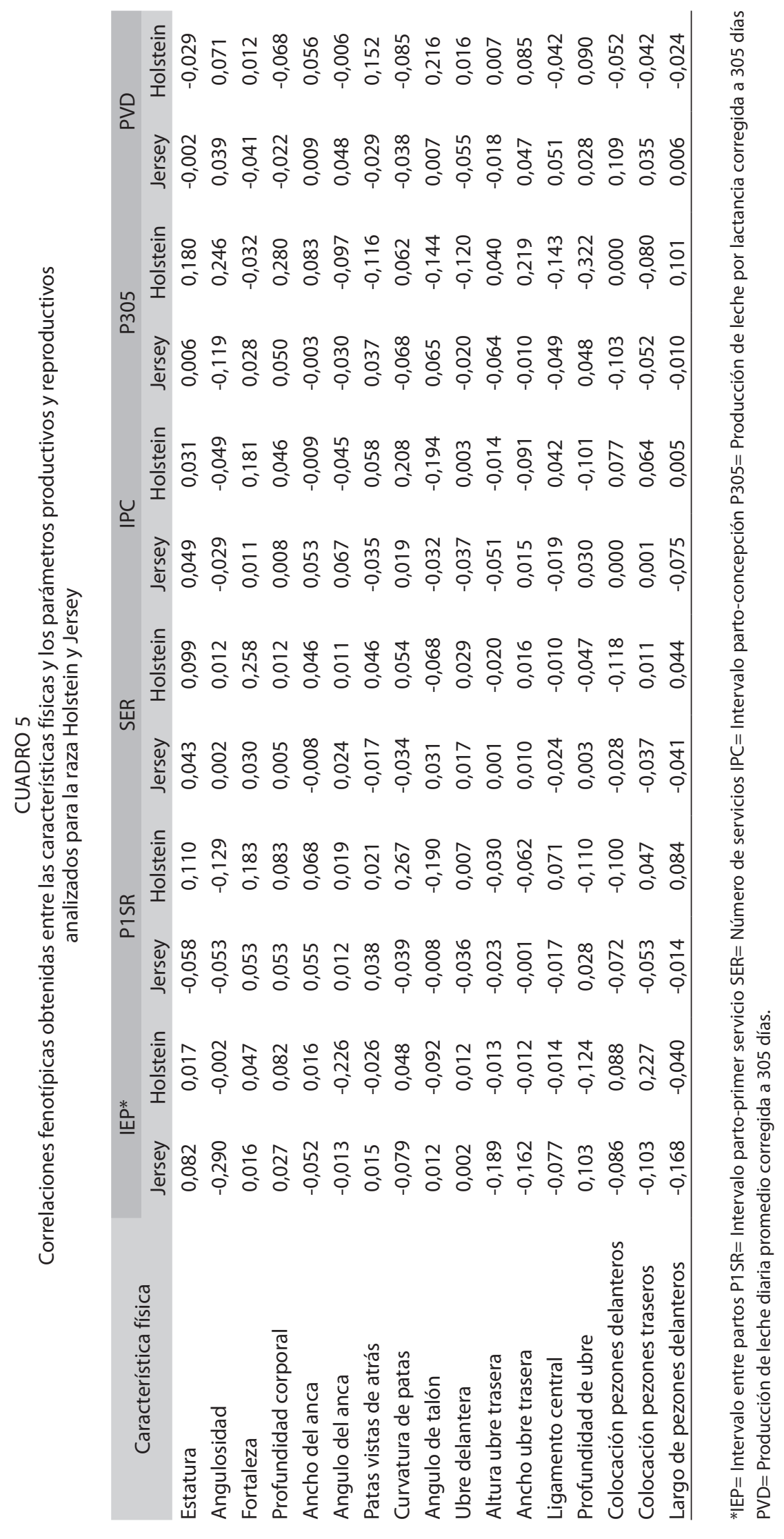


CUADRO 6

Características físicas de cuerpo y su influencia en la reproducción y producción de las vacas Holstein y Jersey

\begin{tabular}{|c|c|c|c|c|c|c|c|c|c|c|c|c|c|}
\hline \multirow{2}{*}{ Característica } & \multirow{2}{*}{ Rango } & \multicolumn{2}{|c|}{ IEP (Días) } & \multicolumn{2}{|c|}{ P1SR (Días) } & \multicolumn{2}{|c|}{ IPC (Días) } & \multicolumn{2}{|c|}{ P305 (kg) } & \multicolumn{2}{|c|}{ SER } & \multicolumn{2}{|c|}{ PVD (kg) } \\
\hline & & Jersey & Holstein & Jersey & Holstein & Jersey & Holstein & Jersey & Holstein & Jersey & Holstein & Jersey & Holstein \\
\hline \multirow[t]{3}{*}{ Estatura } & 1 & 375,4 & 401,4 & 68,9 & 70,7 & 80,9 & 95,6 & $6266,3^{b}$ & 8126,2 & $1,7^{\mathrm{a}}$ & 1,6 & 25,2 & 20,1 \\
\hline & 2 & 384,1 & 388,6 & 68,7 & 83,3 & 80,4 & 96,0 & $6934,7^{\mathrm{a}}$ & 8380,1 & $2,0^{\mathrm{ab}}$ & 2,1 & 25,1 & 29,8 \\
\hline & 3 & 388,7 & 390,0 & 69,5 & 87,7 & 84,7 & 102,0 & $7186,0^{a}$ & 9104,1 & $2,4^{b}$ & 1,8 & 26,0 & 28,7 \\
\hline \multirow{3}{*}{$\begin{array}{l}\text { Carácter } \\
\text { lechero }\end{array}$} & 1 & 383,3 & 397,0 & 67,8 & 91,6 & 78,6 & 103,0 & $6711,5^{b}$ & $8272,5^{b}$ & 2,0 & 1,7 & $23,9^{a b}$ & $24,9^{b}$ \\
\hline & 2 & 382,6 & 384,0 & 68,4 & 80,9 & 80,2 & 95,3 & $6869,11^{\text {ba }}$ & $8361,5^{b}$ & 2,0 & 2,2 & $26,2^{\mathrm{a}}$ & $32,5^{\mathrm{a}}$ \\
\hline & 3 & 386,9 & 395,6 & 72,1 & 83,1 & 87,3 & 99,8 & $7159,9^{a}$ & $9543,7^{\mathrm{a}}$ & 2,2 & 1,8 & $23,4^{b}$ & $25,3^{b}$ \\
\hline \multirow[t]{3}{*}{ Fortaleza } & 1 & $372,8^{\mathrm{a}}$ & $386,9^{\mathrm{ab}}$ & 66,8 & 77,8 & 76,9 & 88,9 & $6585,8^{b}$ & 8683,5 & 1,9 & $1,4^{\mathrm{a}}$ & 25,1 & 29,5 \\
\hline & 2 & $396,9^{b}$ & $364,0^{\mathrm{a}}$ & 68,5 & 82,1 & 81,5 & 84,5 & $7199,3^{a}$ & 7817,7 & 2,0 & $3,8^{b}$ & 24,8 & 24,8 \\
\hline & 3 & $385,4^{b}$ & $397,3^{b}$ & 70,8 & 89,7 & 84,3 & 108,7 & $6971,2^{\mathrm{a}}$ & 8686,6 & 2,1 & $1,8^{\mathrm{ab}}$ & 25,5 & 30,3 \\
\hline \multirow{3}{*}{$\begin{array}{l}\text { Profundidad } \\
\text { Corporal }\end{array}$} & 1 & $371,9^{a}$ & 384,3 & 72,9 & 81,7 & 72,9 & 94,6 & $6155,0^{c}$ & $8083,2^{b}$ & $2,0^{\mathrm{a}}$ & 1,5 & 24,1 & 29,8 \\
\hline & 2 & $381,0^{\mathrm{a}}$ & 389,4 & 81,5 & 84,3 & 81,5 & 99,3 & $6882,5^{b}$ & $8946,4^{b}$ & $2,0^{\mathrm{a}}$ & 2,2 & 25,8 & 30,3 \\
\hline & 3 & $402,5^{b}$ & 399,0 & 84,5 & 85,1 & 84,5 & 97,3 & $7360,7^{a}$ & $9712,3^{\mathrm{a}}$ & $2,5^{\mathrm{b}}$ & 1,9 & 23,2 & 25,0 \\
\hline \multirow[t]{3}{*}{ Ángulo anca } & 1 & 384,6 & 409,3 & 66,9 & $87,8^{b}$ & 80,6 & $107,6^{b}$ & $6848,6^{b}$ & 9056,1 & 2,0 & $1,5^{\mathrm{a}}$ & 25,8 & 28,7 \\
\hline & 2 & 395,8 & 409,1 & 69,3 & $60,2^{\mathrm{a}}$ & 82,3 & $69,9^{\mathrm{a}}$ & $7599,6^{\mathrm{a}}$ & 8448,0 & 2,3 & $4,3^{b}$ & 26,2 & 29,1 \\
\hline & 3 & 382,0 & 383,9 & 68,9 & $85,2^{b}$ & 80,8 & $98,6^{\mathrm{ab}}$ & $6792,0^{b}$ & 8536,7 & 2,0 & $1,6^{\mathrm{a}}$ & 25,1 & 29,6 \\
\hline
\end{tabular}

IEP= Intervalo entre partos $\mathrm{P} 1 \mathrm{SR}=$ Intervalo parto-primer servicio SER= Número de servicios IPC= Intervalo parto-concepción P305= Producción de leche por lactancia corregida a 305 días PVD= Producción de leche diaria promedio corregida a 305 días DA=días abiertos. Letras diferentes en una misma columna para cada fila, presentan diferencias significativas $(p<0,05)$.

\section{Relación de las características de patas y pezuñas} sobre las variables productivas y reproductivas en animales Jersey y Holstein: con la información contenida en el Cuadro 8, se nota que las patas traseras vistas desde atrás tanto en animales Jersey como en Holstein es una característica del componente de patas que afecta la producción y reproducción de las vacas, donde animales que presenten patas que no estén ni juntas (uno a tres) ni separadas (siete a nueve), presentan una tendencia a registrar una mayor producción de leche corregida a 305 días y requieren una menor cantidad de días para registrar un parto.

Características físicas que afectan de forma directa el intervalo entre partos (IEP): para la raza Jersey, las características físicas que más influencia tienen sobre el IEP son la estatura, las patas vistas de atrás, la ubre delantera y la altura de la ubre (Cuadro 9). En el caso de la ubre, estas variables disminuyen el intervalo entre partos en 4,20 y 3,67 días. En cambio, para la raza Holstein (Cuadro $10)$, se consideran el ángulo del anca, ligamento central y la colocación de los pezones traseros, donde mayores puntuaciones en las ancas y en el ligamento central de la ubre reducen los días entre parto en 6,75 y 7,22 días.

Características físicas que afectan de forma directa la producción de leche en una lactancia corregida a 305 días: en los Cuadros 11 y 12, se describen las variables físicas que influyen de forma significativa sobre la producción de leche para animales de la raza Holstein y Jersey respectivamente, donde se da una tendencia de las variables a conformar los componentes corporales y de ubre del semoviente. Un mayor puntaje en la característica de angulosidad de los animales, genera un efecto positivo en la producción de leche por lactancia en animales Holstein, mientras que, en animales Jersey, este efecto es negativo. En el caso de la profundidad corporal, el efecto de una mayor puntuación, genera un aumento en la producción de leche de 1,85 veces en animales Holstein que en animales Jersey. En el caso de altas calificaciones en el componente de ubres de las razas evaluadas, genera una reducción en la producción de leche en cada lactancia. 
CUADRO 7

Características físicas de conformación de ubres y su influencia en la reproducción y producción de las vacas Holstein y Jersey

\begin{tabular}{|c|c|c|c|c|c|c|c|c|c|c|c|c|c|}
\hline \multirow{2}{*}{ Característica } & \multirow{2}{*}{ Rango } & \multicolumn{2}{|c|}{ IEP (Días) } & \multicolumn{2}{|c|}{ P1SR (Días) } & \multicolumn{2}{|c|}{ IPC (Días) } & \multicolumn{2}{|c|}{ P305 (kg) } & \multicolumn{2}{|c|}{ SER } & \multicolumn{2}{|c|}{ PVD (kg) } \\
\hline & & Jersey & Holstein & Jersey & Holstein & Jersey & Holstein & Jersey & Holstein & Jersey & Holstein & Jersey & Holstein \\
\hline \multirow{3}{*}{$\begin{array}{l}\text { Ubre } \\
\text { delantero }\end{array}$} & 1 & 379,2 & 393,5 & 68,2 & 83,1 & 81,1 & 97,0 & $6710,5^{b}$ & 8906,9 & 2,1 & 2,0 & 24,4 & 29,4 \\
\hline & 2 & 390,2 & 393,3 & 68,1 & 76,0 & 73,3 & 87,2 & $7512,2^{a}$ & 8682,8 & 2,1 & 1,7 & 25,6 & 25,8 \\
\hline & 3 & 385,0 & 386,9 & 69,3 & 85,6 & 82,2 & 100,4 & $6870,3^{b}$ & 8413,3 & 2,0 & 2,0 & 25,7 & 30,3 \\
\hline \multirow{3}{*}{$\begin{array}{l}\text { Altura ubre } \\
\text { trasera }\end{array}$} & 1 & 391,4 & 387,9 & 69,7 & 82,4 & 85,6 & 93,6 & 6857,9 & 8563,2 & $2,4^{b}$ & $1,5^{\mathrm{a}}$ & $23,2^{\mathrm{b}}$ & 30,3 \\
\hline & 2 & 388,4 & 388,2 & 68,3 & 84,6 & 80,4 & 100,9 & 6968,0 & 8416,0 & $2,1^{\mathrm{b}}$ & $2,7^{b}$ & $24,7^{\mathrm{a}}$ & 27,7 \\
\hline & 3 & 376,0 & 393,1 & 69,2 & 83,8 & 80,7 & 97,3 & 6775,8 & 8818,4 & $1,9^{\mathrm{a}}$ & $1,6^{\mathrm{a}}$ & $26,1^{\mathrm{a}}$ & 30,6 \\
\hline \multirow{3}{*}{$\begin{array}{l}\text { Ancho ubre } \\
\text { trasera }\end{array}$} & 1 & $374,6^{\mathrm{a}}$ & 390,5 & 67,8 & $88,2^{b}$ & $73,0^{\mathrm{a}}$ & 104,1 & $6127,5^{b}$ & $8277,2^{\text {ab }}$ & $1,8^{\mathrm{a}}$ & $1,6^{\mathrm{a}}$ & 23,7 & 27,1 \\
\hline & 2 & $397,0^{\mathrm{b}}$ & 300,5 & 67,1 & $65,9^{\mathrm{a}}$ & $74,8^{\mathrm{a}}$ & 100,4 & $7198,3^{a}$ & $8056,7^{b}$ & $1,9^{\mathrm{ab}}$ & $3,5^{b}$ & 25,3 & 32,0 \\
\hline & 3 & $381,2^{\mathrm{a}}$ & 392,1 & 69,7 & $86,4^{b}$ & $85,8^{\mathrm{b}}$ & 100,5 & $7001,8^{a}$ & $8944,0^{\mathrm{a}}$ & $2,1^{b}$ & $1,7^{\mathrm{a}}$ & 25,7 & 30,2 \\
\hline \multirow{3}{*}{$\begin{array}{l}\text { Ligamento } \\
\text { Central }\end{array}$} & 1 & $376,7^{a}$ & 396,6 & 69,8 & 80,2 & 84,1 & 90,8 & $6702,6^{b}$ & $9137,2^{\mathrm{a}}$ & 2,0 & 1,6 & 23,5 & 28,0 \\
\hline & 2 & $393,4^{b}$ & 396,8 & 69,5 & 81,4 & 81,4 & 95,0 & $7243,2^{\mathrm{a}}$ & $8085,6^{b}$ & 2,1 & 1,5 & 25,0 & 32,0 \\
\hline & 3 & $383,4^{\mathrm{ab}}$ & 385,4 & 68,4 & 85,8 & 80,0 & 101,4 & $6857,7^{b}$ & $8474,4^{\mathrm{ab}}$ & 2,0 & 2,2 & 25,7 & 29,4 \\
\hline \multirow{3}{*}{$\begin{array}{l}\text { Profundidad } \\
\text { Ubre }\end{array}$} & 1 & 381,5 & 398,6 & $70,2^{\mathrm{a}}$ & $95,1^{b}$ & 82,5 & $115,9^{b}$ & $7181,1^{b}$ & $9328,0^{a}$ & 1,9 & 1,8 & 24,2 & 27,0 \\
\hline & 2 & 395,9 & 381,6 & $68,7^{\mathrm{b}}$ & $63,7^{\mathrm{a}}$ & 80.8 & $62,4^{\mathrm{a}}$ & $7701,7^{a}$ & $8933,6^{a b}$ & 2,0 & 1,5 & 23,9 & 29,3 \\
\hline & 3 & 381,8 & 388,2 & $68,3^{a}$ & $83,6^{b}$ & 80,4 & $98,0^{\mathrm{b}}$ & $6567,6^{c}$ & $8288,5^{b}$ & 2,1 & 2,1 & 25,8 & 30,2 \\
\hline \multirow{3}{*}{$\begin{array}{l}\text { Colocación } \\
\text { Pezones } \\
\text { Delanteros }\end{array}$} & 1 & 372,4 & 395,8 & 70,2 & 92,1 & 80,0 & 108,3 & $6685,6^{b}$ & 8730,8 & 2,0 & 2,0 & 30,1 & $23,7^{b}$ \\
\hline & 2 & 385,1 & 386,8 & 67,1 & 81,4 & 76,6 & 95,1 & $7520,2^{\mathrm{a}}$ & 8953,6 & 2,0 & 1,7 & 26,9 & $26,7^{\mathrm{a}}$ \\
\hline & 3 & 386,8 & 388,7 & 68,7 & 81,9 & 81,8 & 95,4 & $6836,0^{b}$ & 8471,8 & 2,0 & 2,0 & 29,9 & $25,4^{\mathrm{ab}}$ \\
\hline \multirow{3}{*}{$\begin{array}{l}\text { Colocación } \\
\text { Pezones } \\
\text { Traseros }\end{array}$} & 1 & 386,2 & $373,1^{\mathrm{ab}}$ & 71,1 & $79,5^{\mathrm{ab}}$ & 83,9 & 88,0 & $6900,1^{b}$ & 8931,9 & 2,2 & $1,5^{\mathrm{a}}$ & 30,7 & $22,5^{b}$ \\
\hline & 2 & 391,2 & $365,5^{\mathrm{a}}$ & 68,0 & $68,0^{\mathrm{a}}$ & 77,8 & 92,4 & $7312,0^{a}$ & 8133,4 & 2,0 & $4,2^{b}$ & 32,9 & $25,3^{\mathrm{a}}$ \\
\hline & 3 & 378,5 & $401,2^{b}$ & 68,7 & $87,9^{b}$ & 81,8 & 102,5 & $6637,6^{b}$ & 8538,0 & 2,0 & $1,6^{a}$ & 28,4 & $25,8^{\mathrm{a}}$ \\
\hline \multirow{3}{*}{$\begin{array}{l}\text { Largo de } \\
\text { Pezones }\end{array}$} & 1 & 391,7 & 386,5 & 66,5 & 82,0 & 80,7 & 97,6 & 6856,7 & 8597,2 & 1,9 & 1,7 & 30,1 & $26,1^{\mathrm{a}}$ \\
\hline & 2 & 382,7 & 392,2 & 68,4 & 85,0 & 80,2 & 100,6 & 6878,5 & 8470,1 & 2,1 & 2,2 & 29,5 & $25,7^{\mathrm{a}}$ \\
\hline & 3 & 380,8 & 388,3 & 71,2 & 82,8 & 82.5 & 92.5 & 6897,6 & 8829,1 & 2,0 & 1,8 & 28,9 & $23,6^{b}$ \\
\hline
\end{tabular}

IEP= Intervalo entre partos $\mathrm{P} 1 \mathrm{SR}=$ Intervalo parto-primer servicio $\mathrm{SER}=$ Número de servicios IPC= Intervalo parto-concepción P305= Producción de leche por lactancia corregida a 305 días PVD= Producción de leche diaria promedio corregida a 305 días.

Letras diferentes en una misma columna para cada fila, presentan diferencias significativas $(p<0,05)$.

CUADRO 8

Características físicas de conformación de patas y pezuñas y su influencia en la reproducción y producción de las vacas Holstein y Jersey

\begin{tabular}{|c|c|c|c|c|c|c|c|c|c|c|c|c|c|}
\hline \multirow{2}{*}{ Característica } & \multirow{2}{*}{ Rango } & \multicolumn{2}{|c|}{ IEP (Días) } & \multicolumn{2}{|c|}{ P1SR (Días) } & \multicolumn{2}{|c|}{ IPC (Días) } & \multicolumn{2}{|c|}{ P305 (kg) } & \multicolumn{2}{|c|}{ SER } & \multicolumn{2}{|c|}{ PVD (kg) } \\
\hline & & Jersey & Holstein & Jersey & Holstein & Jersey & Holstein & Jersey & Holstein & Jersey & Holstein & Jersey & Holstein \\
\hline Patas & 1 & $385,5^{\mathrm{ab}}$ & 388,0 & $71,4^{\mathrm{b}}$ & $88,4^{\text {ba }}$ & 83,9 & $97,0^{\text {ba }}$ & $6850,6^{b}$ & 8658,0 & 2,0 & 2,3 & 25,0 & 28,3 \\
\hline vista & 2 & $389,3^{b}$ & 400,4 & $65,5^{\mathrm{a}}$ & $70,7^{b}$ & 81,5 & $84,6^{b}$ & $7183,2^{a}$ & 8711,9 & 2,1 & 1,4 & 25,2 & 31,8 \\
\hline \multirow[t]{2}{*}{ trasera } & 3 & $376,4^{a}$ & 387,1 & $67,4^{\mathrm{ab}}$ & $89,0^{a}$ & 76,1 & $107,4^{\mathrm{a}}$ & $6715,3^{b}$ & 8443,2 & 2,0 & 1,8 & 25,6 & 30,0 \\
\hline & 1 & 388,1 & 388,4 & 71,0 & $68,9^{b}$ & 81,0 & $78,3^{\mathrm{b}}$ & 6598,9 & 8501,9 & 1,8 & 1,5 & 26,2 & 32,5 \\
\hline \multirow[t]{2}{*}{ Patas vista lateral } & 2 & 384,6 & 383,2 & 68,9 & $85,7^{a}$ & 81,4 & $100,4^{\mathrm{a}}$ & 6938,9 & 8508,1 & 2,0 & 2,3 & 25,6 & 28,4 \\
\hline & 3 & 380,0 & 399,0 & 68,1 & $91,6^{\mathrm{a}}$ & 79,7 & $108,2^{\mathrm{a}}$ & 6808,2 & 8817,4 & 2,0 & 1,7 & 24,0 & 28,2 \\
\hline Ángulo de & 1 & 382,8 & 389,6 & 68,3 & $88,6^{a}$ & 79,0 & $102,4^{a}$ & $6977,9^{a}$ & $8872,6^{a}$ & 2,0 & 2,2 & 25,6 & 27,8 \\
\hline pezuña & 2 & 385,0 & 390,4 & 69,8 & $78,2^{\mathrm{b}}$ & 84,6 & $92,7^{b}$ & $6686,3^{b}$ & $8175,2^{b}$ & 2,1 & 1,7 & 24,4 & 31,5 \\
\hline
\end{tabular}

$\mathrm{IEP}=$ Intervalo entre partos $\mathrm{P} 1 \mathrm{SR}=$ Intervalo parto-primer servicio $\mathrm{SER}=$ Número de servicios $\mathrm{IPC}=$ Intervalo parto-concepción $\mathrm{P} 305=\mathrm{Producción}$ de leche por lactancia corregida a 305 días PVD= Producción de leche diaria promedio corregida a 305 días.

Letras diferentes en una misma columna para cada fila, presentan diferencias significativas $(p<0,05)$. 


\section{CUADRO 9}

Características físicas que influyen en el intervalo entre partos para la raza Jersey

\begin{tabular}{lcccc}
\multicolumn{1}{c}{ Coeficiente } & Estimado estándar & Error & Valor t & Pr $(>|\mathrm{t}|)$ \\
Intercepto & 433,49 & 15,10 & 28,72 & $<2 \mathrm{e}^{-16}$ \\
Estatura & 2,77 & 1,23 & 2,25 & 0,02521 \\
Patas vistas de atrás & 5,74 & 1,18 & 4,85 & $1,7 \mathrm{e}^{-10}$ \\
Ubre delantera & $-4,20$ & 1,22 & $-3,44$ & 0,00063 \\
Altura de la ubre trasera & $-3,67$ & 1,31 & $-2,80$ & 0,00536 \\
\hline
\end{tabular}

CUADRO 10

Características físicas que influyen en el intervalo entre partos para la raza Holstein

\begin{tabular}{lcccc} 
& Coeficiente & Estimado estándar & Valor $t$ & Pr $(>|t|)$ \\
Intercepto & 426,83 & 28,21 & 15,13 & $<2 e^{-16}$ \\
Ángulo del anca & $-6,75$ & 3,12 & $-2,17$ & 0,0320 \\
Ligamento central & $-7,22$ & 2,97 & $-2,43$ & 0,0162 \\
Colocación de los pezones traseros & 8,55 & 2,74 & 3,11 & 0,0022 \\
\hline
\end{tabular}

CUADRO 11

Características físicas que influyen en la producción de leche en una lactancia corregida a 305 días para la raza Holstein

\begin{tabular}{lcccc}
\multicolumn{1}{c}{ Coeficiente } & Estimado estándar & Error & Valor t & Pr $(>|\mathrm{t}|)$ \\
Intercepto & 7817,4 & 917,3 & 8,52 & $1,9 \mathrm{e}^{-14}$ \\
Angulosidad & 207,5 & 101,2 & 2,05 & 0,04225 \\
Profundidad corporal & 200,4 & 104,7 & 1,91 & 0,05757 \\
Ubre delantera & $-198,8$ & 95,0 & -2.09 & 0,03808 \\
Ancho de la ubre trasera & 237,9 & 83,4 & 2,85 & 0,00500 \\
Profundidad de la ubre & $-299,8$ & 79,1 & $-3,79$ & 0,00022 \\
\hline
\end{tabular}

CUADRO 12

Características físicas que influyen en la producción de leche en una lactancia corregida a 305 días para la raza Jersey

\begin{tabular}{lcccc}
\multicolumn{1}{c}{ Coeficiente } & Estimado estándar & Error & Valor t & Pr $(>|t|)$ \\
Intercepto & 7763,8 & 511,5 & 15,18 & $<2 e^{-16}$ \\
Angulosidad & $-101,2$ & 53,5 & $-1,89$ & 0,059 \\
Profundidad corporal & 108,4 & 64,9 & 1,67 & 0,095 \\
Ancho de la ubre trasera & $-80,4$ & 48,1 & $-1,67$ & 0,095 \\
Colocación de pezones delanteros & $-82,3$ & 47,4 & $-1,74$ & 0,083 \\
\hline
\end{tabular}




\section{DISCUSIÓN}

En el caso de la mayor angulosidad en la vaca Jersey esta tiene un efecto negativo en la producción por lactancia a 305 días, mientras que para la raza Holstein el efecto es positivo. En la presente investigación para la raza Holstein la mayor angulosidad favorece la producción de leche al evitar que el animal incremente en peso, al transformar mayor cantidad de los nutrimentos ingeridos en leche aumentando el balance energético negativo. La fortaleza y la producción de leche diaria corregida correlacionan de forma positiva. Lo anterior es congruente con los resultados obtenidos por Hansen, Freeman, y Berger (1983), donde la selección genética para incrementar la producción láctea disminuye la fertilidad, ya que no consumen los nutrimentos necesarios para el nivel de producción. En el caso de la profundidad corporal, las vacas profundas al ser animales más pesados tienen mayor producción en una lactancia, pero también muestran mayor cantidad de problemas reproductivos, y metabólicos debido a la alta producción de leche y mayor peso corporal (Wall, White, Coffey y Brotherstone, 2007).

Según Caraviello et al. (2003), las vacas con ancas altas tienen $15 \%$ más de probabilidad de salir del hato, debido a que provocan una evacuación más lenta de los líquidos, lo que retrasa la recuperación del animal y aumenta la probabilidad de infecciones en el tracto reproductor. En el caso de las variables relacionadas con las patas de los animales y valores de correlación altos, según Laursen, Boelling, y Mark (2009), el efecto directo podría estar dado por la locomoción de los animales y no por un problema de salud o nutricional. Para la raza Jersey, la altura y ancho de la ubre trasera presentó una correlación mayor a 0,15 según Hansen et al. (1983) y Seykora y Mc Daniel (1983), los animales con ubre trasera alta y ancha se asocia con mayor producción de leche a lo largo de una lactancia, y las vacas con mayores producciones de leche tienen intervalos entre partos más largos. Donde las vacas más productoras requieren más servicios para preñarse, y presentan dificultad para detectarle el primer celo después del parto (Ferguson, Galligan, \& Thomsen, 1994). Bajo esta afirmación, Dadati, Keneddy, y Hayes (1986) informan de una correlación de -0,37 entre la ubre trasera y el intervalo entre partos. En el caso de la colocación y forma de los pezones, Zwertvaegher, De Vliegher, Verbist, Van Nuffel, Baert, y Van Nogenberg (2013) informan que una buena posición y forma favorecen la mayor producción de leche, calidad de esta y ubres más saludables.
Las vacas más profundas, más altas y con ancas levemente bajas o niveladas son más productoras ya que son más dóciles y tienen un ordeño más suave y rápido (Berry, Buckley, Dillon, Evans, \& Veerkamp, 2004). A mayor carácter lechero, la vaca produce más leche en su lactancia, debido a que presenta mayor habilidad para mover reservas energéticas corporales (Berry et al., 2004), y presentan una menor conversión alimenticia, por lo que se mantiene con baja condición corporal durante la lactancia (Dechow et al., 2004). En el caso de las vacas Holstein con mayor angulosidad y mayor fortaleza presentaron las mayores producciones en lactancias corregidas a 305 días. Es común que la vaca fuerte no tenga alto puntaje en carácter lechero y no sea una vaca con mucha producción en los tres primeros meses de lactancia por lo que favorece un menor intervalo entre partos en sistemas de confinamiento total, caso contrario, en el sistema de pastoreo implica que las vacas caminen largas distancias y exista mayor competencia por el alimento, por lo que la fortaleza favorece la producción (Zink, Stipkova, \& Lassen, 2011).

El intervalo entre partos es mayor en las vacas Jersey más fuertes y profundas, mientras que las que son altas y tienen cuerpos profundos requieren más servicios para la concepción, debido a que son vacas que por esas características se favorece la mayor producción láctea y tienen correlación genética con la pérdida rápida de peso en la etapa temprana de la lactancia (Berry et al., 2004) por lo que favorece el balance energético negativo. Otro aspecto a considerar es una mala detección de celo, ya que aumenta los servicios necesarios para la preñez. Esta mala detección de celo, se asociaba a una mala práctica del personal, pero se sabe que el balance energético negativo afecta la presentación del celo en forma adecuada, ya que provoca una baja calidad del folículo y el oocito (Van Eerdenburg, Karthaus, Taverne, Merics, \& Szencio, 2002), por lo que la producción de estradiol no es correcta, lo que provoca una expresión de los síntomas del celo más baja de lo normal (Lyimo, Nielen, Ouwltjes, Kruip, \& Van Eerdenburg; 2000). Según Berry et al. (2004) las vacas más profundas tienen menor mérito genético para días al primer servicio, tasa de concepción y requieren mayor cantidad de servicios para lograr una preñez.

Para la raza Holstein, el ángulo del anca es una de las características que presenta diferencia significativa con respecto a la reproducción, de forma específica al número de servicios necesarios para la preñez. Las vacas con ancas niveladas requieren más servicios para lograr una preñez, lo que podría asociarse a una mayor producción 
de leche. Esto no es congruente con lo escrito en la literatura, ya que las vacas con ángulos intermedios tienen menor riesgo de desecho involuntario (que incluye los problemas reproductivos) (Caraviello et al., 2004). Por otra parte, las ancas altas son las que se asocian a problemas reproductivos, ya que los huesos del anca pueden no descender lo suficiente al momento del parto y provocar algún grado de distocia (Cue, Monardes, \& Hayes 1990) y también aumenta la probabilidad de una infección porque la vagina no drena de forma efectiva después del parto (Astiz, González, Ayala, \& Monge, 2002).

Según Somers, Frankena, Noordhuizen-Stassen \& Metz (2003), cerca del 25 al 30\% de las vacas lecheras son tratadas cada año por problemas de patas. Las vacas Jersey con mejores patas vistas de atrás (LRV) tienen menor intervalo entre partos y reciben su primer servicio a menos días que las vacas con problemas en esta característica. En la raza Holstein las patas vistas de atrás (LRV) alteran los días abiertos y los días al primer servicio, cuando la vaca tiene LRV de puntaje alto aumenta los días a primer servicio y los días abiertos. Con respecto a la curvatura lateral se tiene que los animales Holstein con pata más recta tienen menos días post parto necesarios para la concepción. En el caso del ángulo de pezuña los valores bajos son causantes de periodos más largos para el primer servicio; pero en ambas razas los puntajes menores de dicho ángulo se relacionan con mayores producciones en una lactancia corregida a 305 días. La diferencia entre ambas razas en las características de patas y pezuñas se podría relacionar a la diferencia en peso, ya que afecta el desempeño de sus patas, la parte reproductiva y productiva.

En ambas razas, los animales con ángulos bajos de pezuña se relacionan con mayor producción de leche en una lactancia, esto podría relacionarse a que son animales adultos, que presentan desgaste del casco por la edad y al presentar una alta producción de leche sus dietas son altas en alimento balanceado, lo que podría producir una acidosis que desencadena la liberación de sustancias vasoactivas como la endoxina y la histamina, que causan alteraciones en el casco por lo que son animales que presentan cascos largos y con menor ángulo respecto al suelo (Greenough \& Vermunt, 1991).

Es importante considerar que las características patas vistas de atrás, curvatura lateral de las patas y ángulo del casco presentan una baja heredabilidad, por ejemplo, la curvatura lateral de patas tiene una heredabilidad $\left(\mathrm{h}^{2}\right)$ de 0,15; el ángulo de la pezuña un $\mathrm{h}^{2}=0,11$ y la locomoción en general tiene un $\mathrm{h}^{2}=0,11$ (Onyiro \& Brotherstone,
2008). El progreso genético que se puede obtener de una generación a otra es mínimo, ligado a un factor ambiental, como el tipo de piso, la edad, la raza y el momento del recorte funcional (Gilmore, 1978).

Los cuartos traseros llevan la mayor cantidad de leche, por eso la ubre trasera más ancha se relaciona de forma positiva con la producción de leche (Berry et al., 2004). La adecuada conformación de la ubre favorece la producción de leche y la vida productiva de las vacas (Schneider, Durr, Cue, \& Monardes, 2003), un ejemplo es la puntuación intermedia de la ubre delantera con adecuada capacidad y sin largo excesivo formado por tejido adiposo no productivo. Los valores intermedios en el ligamento central son debido a que el peso de una ubre de una vaca alta productora provoca rompimiento del mismo, probablemente el mismo ligamento en una vaca de baja producción sea considerado como muy fuerte, pero por efecto de que sufre menos estrés por el peso de la leche. Otra razón por la que el ligamento central de fortaleza intermedia favorece la adecuada colocación de los pezones, se debe a que si es débil los abre y si es muy fuerte los cierra, lo que provoca problemas con el equipo de ordeño mecánico.

La profundidad de ubre intermedia es una característica que favorece la producción de leche, ya que le proporciona a la ubre adecuada capacidad para almacenar leche, sin sufrir los problemas de salud que presentan las ubres profundas (Pérez-Cabal \& Alenda, 2002). La colocación de los cuatro pezones debajo del cuarto (puntuación intermedia), beneficia a la producción de leche, ya que permite una adecuada succión por parte de las pezoneras, mantiene la adecuada presión en el equipo de ordeño o la adecuada colocación de las pezoneras en el caso de equipos robotizados (Berry et al., 2004). Estas características son de mayor importancia conforme el animal aumenta en número de lactancias, para favorecer su salud, producción y aumentar su permanencia en el hato (Boettcher, Jairath, Koots, \& Dekkers, 1997). Los animales Jersey en producción con ubres traseras altas y anchas, con ligamentos débiles y ubres profundas se asociaron con animales de alta producción de leche (Boettcher et al., 1997). Las vacas altas productoras son más susceptibles a enfermedades y problemas reproductivos debido a que están sometidas a mayor estrés (Jones, Hansen, \& Chester-Jones, 1994). Un animal con un buen sistema mamario tiene buena salud y es de esperar que tenga menos problemas reproductivos, sin embargo, se asocia las buenas ubres con intervalos entre partos más largos 
debido a que ese tipo de ubre se relaciona con mayores producciones a 305 días (Wall et al., 2005).

En cambio, para la raza Holstein, se obtuvo que el ángulo del anca, el ligamento central y la colocación de pezones traseros presenten un efecto. A mayor estatura los IEP son mayores en la raza Jersey. Animales más pesados tienen mayores problemas de patas y mayor requerimiento alimenticio. Hansen, Cole, Marx, y Seykora (1999), informaron que las vacas seleccionadas para ser de mayor talla no presentaron diferencias significativas en producción de leche al compararse con animales más pequeños; sin embargo, las vacas de mayor tamaño tienen menor vida productiva y más problemas reproductivos. La relación negativa entre las características de ubre y el intervalo entre partos, indica que los animales con mejores ubres son animales con IEP más cortos, debido a que las buenas ubres se asocian con menos problemas reproductivos, menos producción de células somáticas y vacas más saludables (Jones et al., 1994). En el caso de las puntas del anca, cuando se ubican más abajo que la cadera, esta posición permite una mayor facilidad de parto y que los movimientos del útero requieran menor tiempo de drenaje de tejidos y de líquidos, lo que ayuda a disminuir el intervalo entre partos (Rutter, 2002).

Las vacas de la raza Holstein, donde las ubres más anchas y menos profundas, tienen influencia positiva en la producción láctea. Pero ambas razas presentaron asociación entre mayor producción de leche y ubres con problemas de conformación (pezones abiertos y ubres delanteras pendulosas) causado por un mayor peso provocado por la cantidad leche o mayor fuerza ejercida sobre los ligamentos de la ubre. Lo obtenido para la raza Holstein en la presente investigación concuerda con lo informado por Sawa, Bogucki, Kresel-Czopek, y Neja (2013) donde reportan de una correlación de 0,14 entre producción de leche en la vida productiva de una vaca y el ancho de ubre trasero. En ese mismo estudio se encontró una correlación negativa entre producción de leche y la ubre delantera, al igual que el resultado obtenido, lo que indica que la alta producción de leche causa daños al ligamento anterior de la ubre, resultado similar al obtenido por Dadpasand, Zamiri, Atashi, y Akhlaghi (2012). Ese daño a la ubre puede ser cuantificado con una correlación de -0,22 de esta característica física y la producción de células somáticas en una lactancia de 305 días, es decir, mejores ubres delanteras producen leche con menos conteo de células somáticas.

En esta investigación se obtuvo una relación negativa entre la profundidad de ubre y la producción de leche en una lactancia, lo cual difiere de varios autores que indican que las vacas con más cantidad de leche tienen ubres más bajas (Norman, Powell, Wright, \& Cassell, 1988). Este estrés provoca en esas vacas altas productoras mayor susceptibilidad a enfermedades y problemas de ubre (Jones et al., 1994), así la importancia de la conformación de las ubres debe ser especial para esas vacas (Boettcher et al., 1997). Esta diferencia en resultados se puede asociar a que las fincas estudiadas tienen descartes rigurosos con respecto a la profundidad de ubre debido a los problemas de salud y calidad de leche que causan las ubre profundas y se combina con planes de mejoramiento genético con énfasis en ubre, por lo que encontrar ubres sobre el corvejón en vacas altas productoras es muy probable, ya que la buena conformación de la ubre es importante para las vacas de mediana y alta producción (Boettcher et al., 1997).

De igual manera que en la raza Jersey, las vacas Holstein con ubres de buena conformación están ligadas a buenas producciones, la ubre trasera ancha, profundidad de ubre intermedia, pezones colocados en el centro de cada cuarto y largo intermedio de los mismos, por otra parte, las vacas de mayor producción se asocian a un ligamento central débil. Este tipo de ubre permite una buena capacidad de almacenamiento y correcta función de los equipos de ordeño, por lo que favorece una mayor producción de leche. El ligamento central más débil que muestran las vacas altas productoras se debe a que la vaca Holstein es la que en promedio produce más leche al día y en una lactancia, este mayor peso de la ubre provoca rompimiento de dicho ligamento, por eso el efecto es mayor en las vacas altas productoras. La elasticidad de este ligamento le permite adaptarse a los cambios de peso por la cantidad de leche, a los movimientos de la vaca y a los cambios por la edad (Smith, 1960). Las vacas Holstein con ubres más profundas demoraron más su primer servicio, al igual que las Jersey la profundidad de ubre se asocia de forma positiva con la producción de leche, por lo que las vacas Holstein altas productoras movilizaron más reservas corporales para cubrir la necesidad de ese nivel productivo en los primeros días de la lactancia (Ferguson, 1991). Esta situación provoca un retraso en la presentación del celo o celo silencioso debido a una limitación en el crecimiento de los folículos ováricos, lo que dificulta la observación del mismo y el retraso al momento de dar la primera inseminación post parto (Henao, 2001).

De los resultados obtenidos, se permite generar un procedimiento de selección apoyado en los modelos 
matemáticos desarrollados, durante la lactancia de animales Holstein y Jersey mediante la evaluación de las variables físicas de los semovientes que permiten agrupar a los animales que mejor se adaptan a las condiciones sanitarias, ambientales, nutricionales y de manejo que el sistema productivo ofrece, con la meta de obtener los animales más eficientes en producción láctea y reproducción.

\section{AGRADECIMIENTOS}

Deseamos agradecer a las fincas productoras de leche de la zona del Volcán Poás que fueron parte de esta investigación al proporcionar toda la información necesaria. También a la empresa Select Sires Inc, por proveer la plataforma tecnológica necesaria para la toma de muestras y a la Universidad de Costa Rica por facilitar las herramientas necesarias para el análisis estadístico y elaboración de esta investigación.

\section{REFERENCIAS}

Astiz, B. S., González, M. J. V., Ayala, L., \& Monge, A. (2002). The influence of the pelvic conformation on the incidence of urovagina an epidemiological study. Proceedings XXII World of Buiatrics Congress, Hanover, Germany.

Berry, D. P., Buckley, F., Dillon, P., Evans, R. D., \& Veerkamp, R. F. (2004). Genetic relationships among linear type traits, milk yield, body weight, fertility and somatic cell count in primiparous dairy cows. Irish Journal of Agricultural and Food Research, 43, 161-176.

Boettcher, P. J. (2005). Breeding for improvement of functional traits in dairy cattle. Italian Journal of Animal Science, 4, 7-16. DOI: 10.4081/ijas.2005.3s.7

Boettcher, P. J., Jairath, L. K., Koots, K. R., \& Dekkers, J. C. M. (1997). Effects of interactions between type and milk production on survival traits of canadian holstein. Journal of Dairy Science, 80, 2984-2995. DOI: 10.3168/jds. S0022-0302(97)76265-9

Caraviello, D. Z., Weigel, K. A., \& Giannola, D. (2004). Analysis of the relationship between type traits and funtional survival in US Holstein catlle using a weibull proportional hazards model. Journal of Dairy Science, 87, 2677-2686. DOI: 10.3168/jds.S0022-0302(04)73394-9

Cue, R. I., Monardes, H. G., \& Hayes, J. F. (1990). Relationships of calving ease with type traits. Journal of Dairy Science, 73, 3586-3590. DOI: 10.3168/jds.S0022-0302(90)79060-1
Dadati, E., Keneddy, B. W., \& Burnside, E. B. (1986). Relationship between conformation and calving interval in Holstein Cows. Journal of Dairy Science, 69, 3112-3119. DOI: 10.3168/jds.S0022-0302(86)80775-5

Dadpasand, M., Zamiri, M. J., Atashi, H., \& Akhlaghi, A. (2012). Genetic relationship of conformation traits with average somatic cell score at 150 and 350days in milk in Holstein cows of Iran. Journal of Dairy Science, 95, 7340-7345. DOI: 10.3168/jds.2011-5002

Dechow, C. D., Rogers, G. W., Klei, L., \& Lawlort, T. J. (2003). Heretabilities and correlations among body condition score, dairy form and selected linear type traits. Journal of Dairy Science, 86, 2236-2242. DOI: 10.3168/jds. S0022-0302(03)73814-4

Dechow, C. D., Rogers, G. W, Sander-Nielsen, U., Klei, L., Lawlor, T. J., Clay, J. S., Freeman, A. E., Abdel-Azim, G., Kuck, A., \& Schnell, S. (2004). Correlations among body condition scores from various sources, dairy form, and cow health from the United States and Denmark. Journal of Dairy Science, 87, 3526-3533. DOI: 10.3168/jds. S0022-0302(04)73489-X

Fatehi, J., Stella, A., Shannon, J. J., \& Boettcher, P. J. (2003). Genetic parameter for feet and leg traits evaluated in different environments. Journal of Dairy Science, 86, 661666. DOI: 10.3168/jds.S0022-0302(03)73644-3

Ferguson, J. D. (1991). Nutrition and reproduction in dairy cows. Veterinary Clinics of North America, 7, 483-507.

Ferguson, J. D., Galligan, D. T., \& Thomsen, N. (1994). Principal descriptors of body condition scoring chart for Holstein dairy cows. Journal of Dairy Science, 77, 2695-2703. DOI: 10.3168/jds.S0022-0302(94)77212-X

Gilmore, J. A. (1978). The effect of housing, age, breed and time after trimming on hoof measurements. Journal of Dairy Science, 61(Suppl.1), 83.

Greenough, P. R., \& Vermunt, J. J. (1991). Evaluation of subclinical laminitis in a dairy herd and observations on associated nutritional and management factors. Veterinary Record, 128, 11-17. DOI: 10.1136/vr.128.1.11

Hansen, L. B., Cole, J. B., Marx, G. D., \& Seykora, A. J. (1999). Productive life and reasons for disposal of Holstein cows selected for large versus small body size. Journal of Dairy Science, 82, 795-801. DOI: 10.3168/jds. S0022-0302(99)75298-7

Hansen, L. B., Freeman, A. E., \& Berger, P. J. (1983). Association of heifer fertility and cow fertility and yield in dairy cattle. Journal of Dairy Science, 66, 306-314. DOI: 10.3168/jds. S0022-0302(83)81789-5

Henao, G. (2001). Reactivación ovárica post parto en bovinos. Universidad Nacional de Colombia. Revista Facultad Nacional Agropecuaria Medellín, 54, 1285-1302. 
Holstein Association USA, INC. (2012). Características descriptivas lineales. Estados Unidos: Holstein Association. Recuperado de http://www.holsteinusa.com/pdf/ print_material/linear_traits_spanish.pdf

Jones, W. P., Hansen, L. B., \& Chester-Jones, H. (1994). Response of health care to selection for milk yield of dairy cattle. Journal of Dairy Science, 77, 3137. DOI: 10.3168/jds. S0022-0302(94)77257-X

Lassen, J., Hansen, M., Sorensen, K., Aamand, G. P., Christensen, L. G., \& Madsen, P. (2003). Genetic relationship between body condition score, dairy character, mastitis, and diseases other than mastitis in first - parity Danish Holstein cows. Journal of Dairy Science, 86, 3730 - 3735. DOI: 10.3168/jds.S0022-0302(03)73979-4

Laursen, M. V., Boelling, D., \& Mark, T. (2009). Genetic parameter for claw and leg health, foot and leg conformation, and locomotion in Danish Holstein. Journal of Dairy Science, 92, 1770-1777. DOI: 10.3168/jds.2008-1388

Lyimo, Z. C, Nielen, M., Ouwltjes, W., Kruip, T. A. M., \& Van Eerdenburg, J. C. (2000). Relationship among estradiol, cortisol, and intensity of estrous behavior in dairy cattle. Theriogenology, 53, 1783-1795. DOI: 10.1016/ S0093-691X(00)00314-9

Mena, M. (2010). Estación 84030, Fraijanes, evaluación desde 1976 hasta el 2010. Instituto Meteorológico Nacional, San José, Costa Rica.

Norman, H. D., Powell, R. L., Wright, J. R., \& Cassell, B. G. (1988). Phenotypic and genetic relationship between linear functional type traits and milk yield $\mathrm{f}$ or five breeds. Journal of Dairy Science, 71, 1880-1896. DOI: 10.3168/jds. S0022-0302(88)79758-1

Onyiro, O. M., \& Brotherstone, S. (2008). Genetic analysis of locomotion and associated conformation traits of holstein-friesian dairy managed in different housing systems. Journal of Dairy Science, 91, 322-328. DOI: 10.3168/jds.2007-0514:

Perez-Cabal, M. A., \& Alendra, R. (2002). Genetic relationships between lifetime profit and type in Spanish Holstein cows. Journal of Dairy Science, 85, 3480-3491. DOI:10.3168/jds. S0022-0302(02)74437-8

Rutter, B. (2002). Puerperio Bovino. Facultad Veterinaria, Universidad de Buenos Aires, Buenos Aires, Argentina.

Sawa, A., Bogucki, M., Krezel-Czopek, S., \& Neja, W. (2013). Relationship between conformation traits and lifetime production efficiency cows. ISRN Veterinary Science, 2013, 1-4. DOI:10.1155/2013/124690
Schneider, M., Durr, J. W., Cue, R. I., \& Monardes, H. G. (2003). Impact of type traits on functional herd life of Quebec Holstein assessed by survival analysis. Journal of Dairy Science, 86, 4083-4089. DOI: 10.3168/jds. S0022-0302(03)74021-1

Sewalem, A., Kistemaker, G. J., Miglior, F., \& Van Doomaal, B. J. (2004). Analysis for the relationship between survival in Canada Holsteins using Weibull Proportional Hazards Model. Journal of Dairy Science, 87, 3938-3946. DOI: 10.3168/jds.S0022-0302(04)73533-X

Seykora, A. J., \& Mc Daniel, B. T. (1983). Heritabilities and correlations of lactation yields and fertility for Holstein. Journal of Dairy Science, 66, 1486-1493. DOI:10.3168/jds. S0022-0302(83)81963-8

Smith, V. (1960). Physiology of lactation. Journal of Dairy Science. 43:1387. doi:10.3168/jds.S0022-0302(60)90333-7

Somers, J. G. C. J, Frankena, K., Noordhuizen-Stassen, E. N., \& Metz, J. H. M. (2003). Prevalence of claw disorders in Dutch dairy cows exposed to several floor systems. Journal of Dairy Science, 86, 2082-2093. DOI: 10.3168/jds. S0022-0302(03)73797-7

Stamschror, J., Seykora, T., \& Hansen, L. (2000). Judging Dairy Cattle. University of Minnesota, Minessota, EE. UU.

Stevenson, J. (2012). What holds A.I. back these days. Hoards Dairymen, 17, 659.

Uggla, E., Jakobsen, J. H., Bergsten, C., Eriksson, J-A., \& Strandberg, E. (2008). Genetic correlations between claw health and feet and leg conformation traits in Swedishdairy cows. Swedish University of Agricultural Sciences, Suiza..

Vacek, M., Stipkova, M., Nemcova, E., \& Bouska, J. (2006). Relationships between conformation traits and longevity of Holstein cows in the Czech Republic. Chez Journal Animal Science, 8, 327-333.

Van Der Waaij, E. H., Holzhauer, M., Ellen, E., Kamphuis, C., \& Jong, G. (2005). Genetic parameters for claw disorders in Dutchdairy cattle and correlations with conformation traits. Journal of Dairy Science, 88, 3672-3678. DOI: 10.3168/jds.S0022-0302(05)73053-8

Van Eerdenburg, F. J. C. M., Karthaus, D., Taverne, M. A. M., Merics, I., \& Szencio, O. (2002). The relationship between estrous behavioral score and time of ovulation in dairy cattle. Journal of Dairy Science, 85, 1150-1156. DOI: 10.3168/jds. S0022-0302(02)74177-5

Wall, E., White, M. S., Coffey, M. P., \& Brotherstone, S. (2005). The relationship between fertility, rump angle, and selected type information in Holstein Friesian cows. 
Journal of Dairy Science, 88, 1521-1528. DOI: 10.3168/jds. S0022-0302(05)72821-6

Wall, E., White, M. S., Coffey, M. P., \& Brotherstone, S. (2007). The relationship between body energy traits and production and fitness traits in first-lactation dairy cows. Journal of Dairy Science, 90, 1527:1537.

Weiss, D., Wenfurtner, M., \& Bruckmaier, R. (2004). Teat anatomy and its relationship in dairy cows. Journal of Dairy Science, 87, 3280-3289. DOI:10.3168/jds.S0022-0302(04)73464-5
Zink, V., Stipkova, M., \& Lassen, J. (2011). Genetic parameters for female fertility, locomotion, body condition score and linear type traits in Czech holstein cattle. Journal of Dairy Science, 94, 5176:5182.

Zwertvaegher, I., De Vliegher, S., Verbist, B., Van Nuffel, A., Baert, J., \& Van Nogenberg, S. (2013). Short communication: Associations between teat dimension and milking-induced changes in teat dimensions and quarter milk somatic cell counts in dairy cows. Journal of Dairy Science 96(2), 1075-1080. DOI: 10.3168/jds.2012-5636 